\title{
Nanomedicinal products: a survey on specific toxicity and side effects
}

\author{
This article was published in the following Dove Press journal: \\ International Journal of Nanomedicine \\ 22 August 2017 \\ Number of times this article has been viewed
}

\author{
Walter Brand ${ }^{1, *}$ \\ Cornelle W Noorlander $1, *$ \\ Christina Giannakou ${ }^{2,3}$ \\ Wim H De Jong ${ }^{2}$ \\ Myrna W Kooi' \\ Margriet VDZ Park ${ }^{2}$ \\ Rob J Vandebriel ${ }^{2}$ \\ Irene EM Bosselaers ${ }^{4}$ \\ Joep HG Scholl ${ }^{5}$ \\ Robert E Geertsma ${ }^{2}$ \\ 'Centre for Safety of Substances \\ and Products, ${ }^{2}$ Centre for Health \\ Protection, National Institute for \\ Public Health and the Environment \\ (RIVM), Bilthoven, ${ }^{3}$ Department of \\ Toxicogenomics, Maastricht University, \\ Maastricht, ${ }^{4}$ Section Pharmacology, \\ Toxicology and Pharmacokinetics, \\ Medicines Evaluation Board \\ (CBG-MEB), Utrecht, ${ }^{5}$ Research \& \\ Analysis Department, Netherlands \\ Pharmacovigilance Centre Lareb, \\ 's-Hertogenbosch, the Netherlands \\ *These authors contributed equally \\ to this work
}

\begin{abstract}
Due to their specific properties and pharmacokinetics, nanomedicinal products (NMPs) may present different toxicity and side effects compared to non-nanoformulated, conventional medicines. To facilitate the safety assessment of NMPs, we aimed to gain insight into toxic effects specific for NMPs by systematically analyzing the available toxicity data on approved NMPs in the European Union. In addition, by comparing five sets of products with the same active pharmaceutical ingredient (API) in a conventional formulation versus a nanoformulation, we aimed to identify any side effects specific for the nano aspect of NMPs. The objective was to investigate whether specific toxicity could be related to certain structural types of NMPs and whether a nanoformulation of an API altered the nature of side effects of the product in humans compared to a conventional formulation. The survey of toxicity data did not reveal nanospecific toxicity that could be related to certain types of structures of NMPs, other than those reported previously in relation to accumulation of iron nanoparticles (NPs). However, given the limited data for some of the product groups or toxicological end points in the analysis, conclusions with regard to (a lack of) potential nanomedicine-specific effects need to be considered carefully. Results from the comparison of side effects of five sets of drugs (mainly liposomes and/or cytostatics) confirmed the induction of pseudo-allergic responses associated with specific NMPs in the literature, in addition to the side effects common to both nanoformulations and regular formulations, eg, with liposomal doxorubicin, and possibly liposomal daunorubicin. Based on the available data, immunotoxicological effects of certain NMPs cannot be excluded, and we conclude that this end point requires further attention.
\end{abstract}

Keywords: adverse effects, drug safety, immunotoxicity, nanomedicine, nanotoxicology, pharmacovigilance

\section{Introduction}

Innovative medical applications of nanotechnology are expected to have a continuously growing impact on health care. ${ }^{1-3}$ These new nanotechnology applications relate to both medicinal products and medical technologies. New applications are becoming available for diagnosis, treatment, monitoring, and prevention of disease. ${ }^{4}$ While the potential advantages such as more effective and less toxic therapeutics and diagnostic interventions are obviously highly desirable, the emergence of nanomedicinal products (NMPs) also gives rise to questions of whether currently used safety assessment procedures provide an adequate evaluation of the quality, safety, and efficacy of these products with regard to any possible nanospecific aspects. ${ }^{5}$ The safety evaluation of NMPs could pose specific challenges associated with the particulate characteristics of their formulations. Especially, the toxicokinetic profile of nanoparticles (NPs), as well as the toxicodynamic effects, can be quite different from that of dissolved chemicals. ${ }^{5}$ It is important to have a thorough understanding of NMPs and their specific properties,

\footnotetext{
Correspondence: Walter Brand Centre for Safety of Substances and Products, National Institute for Public Health and the Environment (RIVM), PO Box I, 3720 BA Bilthoven, the Netherlands

Tel +3I 302743047

Email walter.brand@rivm.nl
} 
not only for regulators and the pharmaceutical industry but also for physicians and pharmacists. ${ }^{5,6}$ Differences in toxicokinetics of nanomedicines compared to conventional, non-nanoformulated medicines may result in marked differences in toxicity and side effects. ${ }^{7}$ The aim of this study was to investigate whether specific toxicity could be related to NMPs, or certain structural types or groups of NMPs, by analyzing the publicly available preclinical toxicity data on approved NMPs. In addition, by comparing several sets of products with the same active pharmaceutical ingredient (API) in a conventional formulation versus a nanoformulation, we aimed to identify whether any side effects observed in humans are specifically associated with the nanoformulation compared to the conventional formulation.

\section{Materials and methods Toxicity data of NMPs}

To gain more insight into the types of toxicity specific for NMPs, the toxicity data of approved NMPs in the EU were systematically analyzed. Therefore, the list of NMPs identified in our previous paper, ${ }^{8}$ which yielded 47 approved NMPs, was extended by an updated Internet and PubMed search up to September 2015 contributing an additional four NMPs approved in Europe. The same search terms were used as reported previously, namely, nanomedicine, nanotechnology, nanodrug, NP, drugs, therapeutics, vaccines, biologicals, diagnostics, pharmaceutics, horizon scan(ning), overview, roadmap, foresight, forecast, future, clinical trials, randomized controlled trials, cohort studies, case reports, human, drug delivery (systems), drug carrier, drug targeting, gene therapy, drug discovery, drug encapsulation, liposomes, micelles, dendrimer, fleximer, hard NP, soft NP, nanodispersion, polymeric NP, protein NP, emulsion, virosome, and any combination of the mentioned terms. ${ }^{8}$ The resulting 51 NMPs included 22 formulations with nanocarriers (liposomes, polymer conjugates, polymeric NPs, micelles, and a gene therapy product) and 29 nanosized APIs (nAPIs). In the current paper, these were further classified into categories with respect to structure and drug type (Table 1): cytostatics (9), formulations with nanocarriers (11), formulations with nAPIs (9), monoclonal antibodies/recombinant enzymes and proteins (17), vaccines (4), and one gene therapy product (1). As for certain products, their pharmacotherapeutic use is closely related to their safety evaluation and/or toxicity, cytostatics, monoclonal antibodies/recombinant enzymes and proteins, and vaccines, and the single gene therapy products are grouped separately from the (other) formulations with nanocarriers and formulations with nAPIs (Table 1). A considerable part of the identified NMPs consists of monoclonal antibodies/recombinant enzymes or proteins. As explained earlier in Noorlander et $\mathrm{al},{ }^{8}$ such protein NPs comply with the description used for NMPs: although their sizes are generally not available, it can be assumed that they are particulate substances of less than $1,000 \mathrm{~nm} .{ }^{8}$ In addition, they have been designed to have specific properties to get the intended functionality. However, they are well-known separate categories of biological products with their own related potential toxicity and therefore not further discussed in this paper. ${ }^{9}$ Similarly, also, vaccines and gene therapy products have their own specific properties and are not expected to lead to additional nanospecific toxicity either. Therefore, also, vaccines and gene therapy products are not further discussed in this paper either.

For the remaining 29 products, when present, the publicly available, preclinical information on toxicology, usually from the assessment reports or scientific discussion documents by European Medicines Agency (EMA), was analyzed for data on the following classes of end points of toxicology: acute toxicity (ie, single-dose studies), subacute toxicity (ie, repeated-dose studies or [semi]chronic toxicity studies), genotoxicity, carcinogenicity, reproductive toxicity, developmental toxicity, and immunotoxicity. All products were marked individually (Supplementary materials), and per structural type and group of drugs of interest (Table 2) with "positive" (effect), "negative" (no effect), or "unknown." The latter was used in case no information was available, ie, no assay for the respective end point had been performed or the performance, outcome, or results were not reported. Sometimes, an assay for a specific end point was not performed because reference was made to the known characteristics of the API, or a related non-nanoformulation (not performed; with reference), or waived because a scientific opinion was expected to be published altering the set of registration guidance documents requirements (eg, because of the concept paper recommending revising the existing guideline on performing single-dose/acute toxicity test in addition to the repeated-dose toxicity studies ${ }^{10}$ ). All these situations are classified as "unknown" in Table 2. The toxicity data per product, the connection of brand name and product identification number, the API, and the public source of information are provided in the Supplementary materials.

\section{Side effects of NMPs}

To gain more insight into the side effects of NMPs, a comparison was made between the side effects, in humans, of 
Table I Products resulting from the inventory of registered NMPs within the EU, ordered by the type of nanostructure or drug

\begin{tabular}{|c|c|c|c|}
\hline Structure type & Type of drug & $\begin{array}{l}\text { Number of } \\
\text { products }\end{array}$ & Product brand names $^{a}$ \\
\hline \multicolumn{4}{|l|}{ Cytostatics } \\
\hline \multirow[t]{2}{*}{ Liposomes } & & 6 & Caelyx, DepoCyt, DaunoXome, ${ }^{b}$ Eloxatin, ${ }^{b}$ \\
\hline & & & Mepact, Myocet \\
\hline Micelle & & I & Taxotere \\
\hline Protein & & I & Alimta \\
\hline Others & & I & Abraxane \\
\hline \multicolumn{4}{|c|}{ Formulations with nanocarriers } \\
\hline \multirow[t]{2}{*}{ Liposomes } & Antifungal agent & 2 & Abelcet, ${ }^{b}$ AmBisome \\
\hline & Ophthalmological & I & Visudyne \\
\hline Polymer & Recombinant protein conjugated to PEG & 3 & Mircera, Pegasys, Pegintron \\
\hline \multirow[t]{3}{*}{ conjugates } & Recombinant protein & 2 & Neulasta, Somavert \\
\hline & Phosphate-binding agent & I & Renagel \\
\hline & Oligonucleotide & I & Macugen \\
\hline Polymeric NP & Antipsychotic & I & Risperdal consta \\
\hline \multicolumn{4}{|c|}{ Formulations with nAPIs } \\
\hline \multirow[t]{2}{*}{ Iron NPs } & Imaging agent & 2 & Lumirem, ${ }^{\mathrm{b}, \mathrm{c}}$ Endorem ${ }^{\mathrm{b}, \mathrm{c}}$ \\
\hline & Antianemic & 2 & Rienso, Venofer \\
\hline \multirow[t]{3}{*}{ Nanodispersions } & Antiemetic & I & Emend \\
\hline & Immunosuppressant & I & Rapamune \\
\hline & Antipsychotic & I & Invega \\
\hline Emulsion & Immunosuppressant & I & Neoral \\
\hline Protein & Antifungal agent & I & Cancidas \\
\hline \multicolumn{4}{|c|}{ Monoclonal antibody/recombinant enzyme and proteins } \\
\hline & Monoclonal antibody & 13 & Adcetris, Arzerra, Avastin, Erbitux, \\
\hline & & & Vectibix, Herceptin, Lemtrada, Mabthera, \\
\hline & & & Orencia, Remicade, Xolair, Yervoy, Zevalin \\
\hline & Recombinant enzyme, protein, human protein & 4 & Aldurazyme, Avonex, Fasturtec, Nanocoll ${ }^{b}$ \\
\hline \multicolumn{4}{|c|}{ 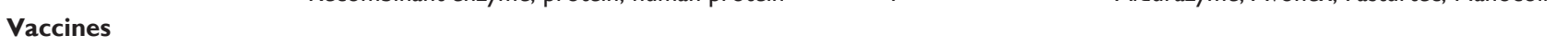 } \\
\hline & Emulsion & I & Pandemrix \\
\hline & Polymeric NP & I & Focetria \\
\hline & Others & 2 & Cervarix, Gardasil \\
\hline \multicolumn{4}{|l|}{ Gene therapy } \\
\hline & & I & Glybera $^{\mathrm{b}}$ \\
\hline
\end{tabular}

Notes: During grouping, cytostatics, monoclonal antibodies/recombinant enzymes and proteins, vaccines, and gene therapy have been allocated to separate categories

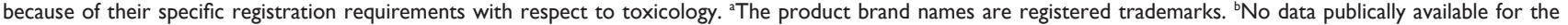
product. 'Withdrawn from market.

Abbreviations: nAPI, nanosized active pharmaceutical ingredient; NMPs, nanomedicinal products; NP, nanoparticle; PEG, polyethylene glycol.

NMPs versus conventional products with the same API. Five sets of drugs, each consisting of one conventional product and one NMP, were chosen for the comparison of the side effects (Table 3). Information for such a comparison of a conventional form and a nanoform was available for those five sets only. Product information sheets from the EMA and the Dutch pharmacotherapeutical compendium, as well as available summaries of product characteristics, were used for the investigation of the side effects. The MedDRA (Medical Dictionary for Regulatory Activities) dictionary of terms was used to classify the side effects of the drugs (MedDRA system organ class [SOC]). The side effects of the traditional product and the NMP were compared, and only the differences are presented. Besides the classification, the frequency of the side effects was also presented: "very common" ( $\geq 1 / 10)$, "common" $(\geq 1 / 100$ to $<1 / 10)$, "uncommon" $(\geq 1 / 1,000$ to $<1 / 100)$, "rare" ( $\geq 1 / 10,000$ to $<1 / 1,000)$, "very rare" $(<1 / 10,000)$, or "unknown" whenever the frequency cannot be estimated from the available data. Note that differences in the application of products, eg, differences in the diseases treated, could potentially greatly affect the reported side effects.

In addition to the data on these side effects retrieved from the product information, the database of the Netherlands Pharmacovigilance Centre Lareb, which collects and analyzes spontaneous reports of suspected adverse reactions of medicines, was searched for case reports on the five sets of drugs. It should be noted that a report in the Lareb 


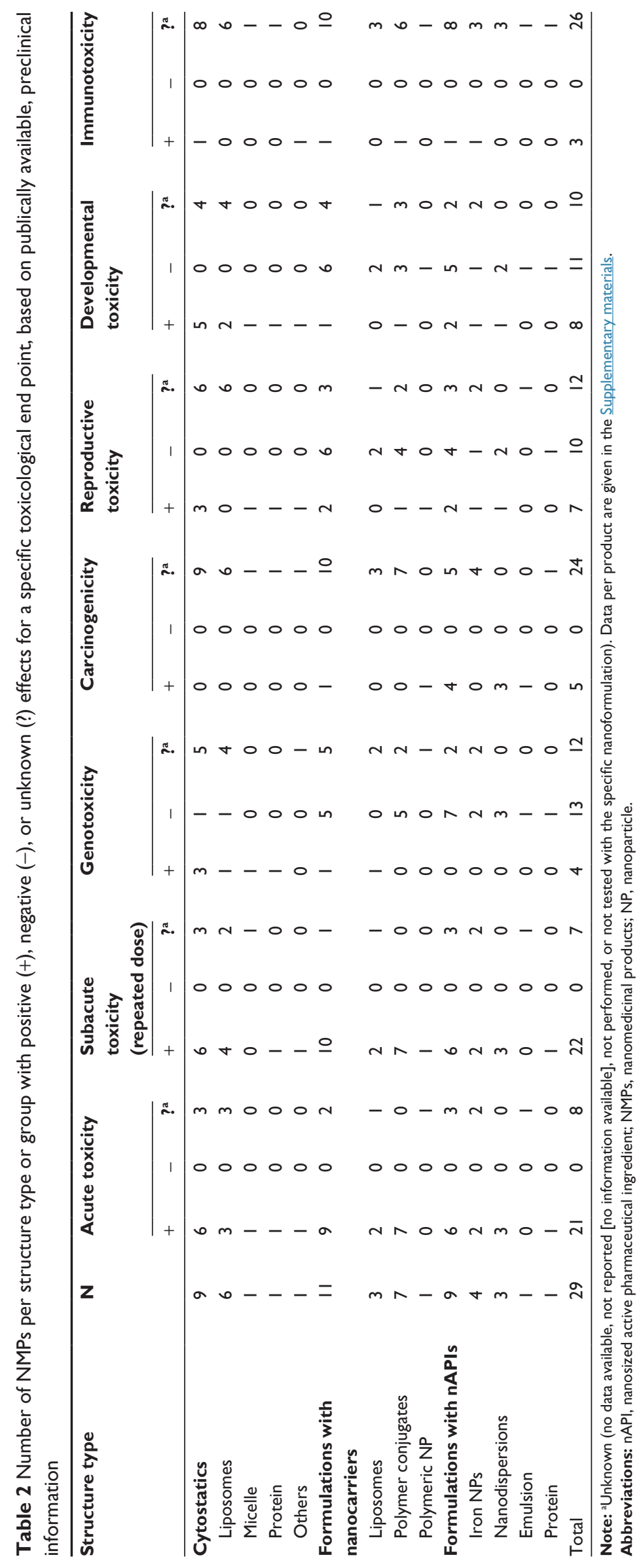


Table 3 Overview of the sets of drugs investigated for the comparison of the side effects

\begin{tabular}{|c|c|c|c|c|}
\hline $\begin{array}{l}\text { Active } \\
\text { substance }\end{array}$ & $\begin{array}{l}\text { Brand name } \\
\text { (conventional drug) }\end{array}$ & $\begin{array}{l}\text { Nanoformulation of } \\
\text { active substance }\end{array}$ & $\begin{array}{l}\text { Brand name } \\
\text { (nanosized drug) }\end{array}$ & Application \\
\hline Paclitaxel & $\operatorname{Taxol}^{\circledR}$ & Nab-paclitaxel & Abraxane $^{\circledR}$ & $\begin{array}{l}\text { Cancer treatment, } \\
\text { chemotherapy }\end{array}$ \\
\hline Amphotericin B & Fungizone $^{\circledR}$ & $\begin{array}{l}\text { Liposomal } \\
\text { amphotericin B }\end{array}$ & AmBisome ${ }^{\circledR}$ & $\begin{array}{l}\text { Infectious diseases, } \\
\text { antifungal drug }\end{array}$ \\
\hline Doxorubicin & Doxorubicin & $\begin{array}{l}\text { Pegylated liposomal } \\
\text { doxorubicin }\end{array}$ & Caelyx ${ }^{\circledR}$ & $\begin{array}{l}\text { Cancer treatment, } \\
\text { chemotherapy }\end{array}$ \\
\hline Daunorubicin & Cerubidine $^{\circledR}$ & $\begin{array}{l}\text { Liposomal } \\
\text { daunorubicin }\end{array}$ & DaunoXome ${ }^{\circledR}$ & $\begin{array}{l}\text { Cancer treatment, } \\
\text { chemotherapy }\end{array}$ \\
\hline Cytarabine & Cytarabine & Liposomal cytarabine & DepoCyt ${ }^{\circledR}$ & $\begin{array}{l}\text { Cancer treatment, } \\
\text { chemotherapy }\end{array}$ \\
\hline
\end{tabular}

database does not necessarily imply a causal relationship between the complaint and the medicine. It is to be regarded as a suspicion of the reporter that the medicine might be involved in the complaints. In addition, due to the nature of spontaneous reporting, no incidence rates can be calculated, and comparisons between drugs should be made with much caution. Still, these data could provide more insight with respect to the nature and severity of the side effects in addition to the frequency reported by the EMA, the Dutch pharmacotherapeutical compendium, and the summaries of product characteristics. The detailed results from this search are provided in the Supplementary materials.

\section{Results}

The 29 NMPs were marked individually (Supplementary materials), and per structure type and group of drugs of interest (Table 2), for information on specific end points of toxicology based on publicly available, preclinical toxicity information. The outcome for the specific end points was marked with "positive" (effect), "negative" (no effect), or "unknown," according to the criteria mentioned in the "Materials and methods" section.

Some toxicological effects greatly depend on the altered pharmacokinetics of NMPs compared to conventional, non-nanoformulated medicines. Differences in absorption, distribution, metabolism, or excretion (ADME) factors, or different interactions, could for instance result in altered availability on certain cites or within specific tissues of the body. These aspects may result in differences in toxicity and side effects of NMPs as well. ${ }^{7}$

\section{Toxicity data of NMPs}

Although the group sizes in Table 2 are small, with a total of 29 products divided in three diverse groups, still the following notes can be made on the outcome of the survey on toxicity specific for certain groups and structural types of
NMPs, ie, cytostatics, formulations with nanocarriers, and formulations with nAPIs.

\section{Cytostatics}

Cytostatics, a drug-type group containing nine products, were regarded separately and showed almost no negative and many positive responses for specific end points in preclinical toxicology (Table 2). The effects of these products are unlikely to be directly related to the nanocarrier or nanoformulation, but to the APIs, which are intended to be very toxic to cancer cells. The respective nanoformulations, mostly liposomes, were often designed to lower their toxicity to healthy tissues as well as to increase their efficacy. The use of nanoformulations of cytostatics sometimes resulted in a change in toxicological profile. For example, a liposomal encapsulation of doxorubicin with surface-bound methoxypolyethylene glycol $\left(\right.$ Caelyx $\left.^{\circledR}\right)$ is known to be less cardiotoxic and nephrotoxic than unbound doxorubicin; however, it produces more dermal lesions primarily on the feet and legs. ${ }^{11}$ A liposome-encapsulated doxorubicin-citrate complex $\left(\right.$ Myocet $^{\mathbb{R}}$ ) shows less cardiotoxicity than unbound doxorubicin; however, it induces an increased bone marrow suppression. These examples of a shift in toxicological profile due to changes in the pharmacokinetics of drugs could still reduce the overall toxicity of a drug, the severity of the side effects, or better suit specific patients. ${ }^{12}$ A liposomal formulation of cytarabine (DepoCyt ${ }^{\circledR}$ ) has been reported to cause the same efficacy but less toxicity in the treatment of specific cancers compared to unbound cytarabine, ${ }^{13,14}$ and a liposomal formulation of daunorubicin citrate $\left(\right.$ DaunoXome ${ }^{\circledR}$ ) is associated with reduced cardiotoxicity compared to conventional daunorubicin. ${ }^{15}$ In addition to the six liposomal encapsulations, other structures of nanocarriers are applied, such as docetaxel solubilized by micelle formation with polysorbate 80 (Taxotere $^{\circledR}$ ), proteinbound pemetrexed (Alimta ${ }^{\circledR}$ ), or albumin-bound paclitaxel $\left(\right.$ Abraxane $\left.^{\circledR}\right)$. In the latter, paclitaxel is bound to albumin 
as a delivery vehicle to circumvent solvent-related toxicity issues such as hypersensitivity and neuropathy arising from the conventional administration of paclitaxel. ${ }^{16,17}$ In addition, for these types of nanoformulations, the toxic effects of the products are likely related to the API rather than to the specific nanocarrier, or not tested because of the known effects of the API or the intended use of the products.

Cytostatics contain known carcinogenic substances (doxorubicin, cytarabine), which is considered acceptable in view of the intended use (ie, the treatment of cancer), and therefore, carcinogenicity testing has been waived for the liposomal doxorubicin and liposomal cytarabine formulations. Similarly, reproductive toxicity and developmental toxicity testing has been waived because of the known reproductive toxicity, teratogenicity, or developmental effects of, eg, doxorubicin and cytarabine. Of the nine cytostatics, of only albumin-bound paclitaxel the immunotoxicity has been assayed specifically.

\section{Formulations with nanocarriers}

Of the 11 products that can be classified as formulations with nanocarriers (other than cytostatics), the acute and subacute toxic effects are often also related to the API. This accounts especially for the liposomal formulations, as these were designed to lower toxicity as well as to increase efficacy. For instance, an encapsulation of amphotericin B into liposomes $\left(\right.$ AmBisome $^{\circledR}$ ) is used to minimize nephrotoxicity associated with amphotericin. All seven polymer conjugates among the formulations with nanocarriers show acute and subacute toxicity, albeit generally to a low degree. Apart from methoxypolyethylene glycol epoetin beta $\left(\right.$ Mircera $\left.^{\circledR}\right)$, an antianemic for anemia associated with chronic kidney disease, the polymer conjugates are well tolerated, only giving acute and repeated-dose effects at relatively high doses. These toxic doses are far beyond the intended clinical doses, which show mild effects with little clinical significance. The acute and subacute toxicity of methoxypolyethylene glycol epoetin beta is associated with exaggerated pharmacological effects not expected to be caused by the polymer itself.

With regard to genotoxicity, the light-dependent effect of the ophthalmological drug verteporfin (Visudyne ${ }^{\circledR}$ ) on DNA found is not expected to occur in patients. This is because verteporfin concentrates in the cytoplasm rather than the nucleus, and singlet oxygen has a very short half-life and diffusion path. ${ }^{18}$

In addition, with formulations with nanocarriers, testing was sometimes waived because of the known (or suspected) carcinogenicity of the API (eg, pegvisomant), or immunotoxicity, teratogenicity, or developmental effect of the API (eg, peginterferon alfa 2a, pegvisomant, and peginterferon alfa $2 b$ ).

Of the 11 products classified as formulations with nanocarriers, the immunotoxicity of only the polymer conjugate pegvisomant, a recombinant protein, has been assayed specifically.

\section{Formulations with nAPIs}

The nine other products that were classified as formulations with nAPIs formed a very diverse group of products. Of the four iron NP products included, half of them showed both acute and subacute toxicity. There are toxicity concerns for iron oxide NPs because of oxidative stress, unpredictable cellular responses, induction of signaling pathways, and iron overload. ${ }^{19,20}$ Indeed, with regard to the two antianemics among the iron NP structure type subgroup (Table 2), the toxicity seen at high doses of ferumoxytol (Rienso ${ }^{\circledR}$ ) and iron sucrose $\left(V^{2}\right.$ onofer $\left.{ }^{\circledR}\right)$ was associated with accumulation of the iron NPs. Ferumoxytol increased iron blood levels and caused accumulation of iron pigment in multiple tissues was observed including the adrenals, spleen, ovaries, liver, and kidneys, with iron sucrose accumulation resulting in hemosiderosis. For the two nanomedicinal imaging agents, superparamagnetic iron oxide NPs (SPIONs), no toxicity information was publicly available. These two products are withdrawn from the market, mainly due to low demand and high prices. ${ }^{21}$ Interestingly, all three drugs classified as nanodispersions in Table 2 score positive for acute and subacute toxicity and carcinogenicity (but negative for genotoxicity). This apparent similarity in effects of these three different oral nanodispersions, the immunosuppressant sirolimus, the antiemetic aprepitant, and the antipsychotic paliperidone, is most likely not to be explained by the nanodispersion type of the formulation, at least not on the basis of our limited set of data. It seems more likely that the effect of the APIs contained is responsible; these NMPs differ greatly, including in the toxicological profiles, the explanation of carcinogenic potentials and the significance of the effects, or the effects are rather nonspecific (further details are given in the scientific discussions by the European Agency for the Evaluation of Medicinal Products (EMEA), and the respective references are given in the Supplementary materials).

Taken into consideration that, in addition to the limited number of products, the data on several toxicological end points are limited, our survey on toxicity of NMPs does not indicate that certain toxic effects, in terms of specific end points, are associated with specific structural 
types of NMPs - apart from the known iron NP-related toxicity associated with accumulation of the iron NPs. ${ }^{19,20}$ However, such conclusions with regard to potential (lack of) nanomedicine-specific effects need to be considered carefully. When an NMP contains or is composed of a drug with cytostatic activity, many toxic effects were observed including (sub)acute toxicity, genotoxicity, reproductive toxicity, and teratogenicity. These toxic effects can be attributed to the API and are not related to the nanocarrier. For other liposomal preparations, the toxic effects can be attributed to the API rather than to the nanospecific structure of these NMPs as well. In addition, the lack of similarity in toxicity profiles within the various groups or subgroups of NMPs indicates no nanospecific toxic end point.

\section{Side effects of NMP formulations}

In addition to the survey on preclinical toxicity of registered NMPs, we hypothesized that examining the difference in side effects reported in humans between sets of drugs, consisting of the conventional product (non-nano) and the NMP, could possibly help to gain insight into any nanospecific adverse effects. For five NMPs and their API, such a comparison was possible: paclitaxel, amphotericin B, doxorubicin, daunorubicin, and cytarabine (Table 3). Tables 4-8 present an overview of the differences in type or frequency between the observed adverse effects of the conventional API and the nanoformulations. These comparisons do not take into account the severity of the side effects reported. Difference in side effects could originate from differences in toxicology, often caused by altered toxicokinetics. In addition, one should keep in mind that differences in side effects reported sometimes can also originate from different situations in which both products are used, in different treatment regimens or for different indications. In comparisons where this is known to be of importance, this is specifically mentioned.

\section{Nab-paclitaxel versus paclitaxel}

Paclitaxel is one of the most effective chemotherapeutic drugs and is used against a broad range of tumors, such as lung, ovarian, and breast carcinoma. ${ }^{22}$ Due to its low water solubility, paclitaxel can be formulated in a mixture of polyethoxylated castor oil (Cremophor ${ }^{\circledR}$ EL) and dehydrated ethanol. Polyethoxylated castor oil adds to the toxic effects of paclitaxel by producing or contributing to hypersensitivity reactions that commonly occur during infusion. ${ }^{23,24}$ When paclitaxel is bound to albumin as a delivery vehicle, it is called protein-bound paclitaxel or nab (NP albumin-bound) paclitaxel. Both applications are intended for intravenous administration. It is considered that the encapsulation of paclitaxel in biodegradable and nontoxic nano-delivery systems can protect the drug from degradation during circulation and protect the body from toxic side effects of the drug. In addition, there is an increase in circulation half-life and improved pharmacokinetic profile. NMPs also gain efficacy in antitumor activity due to the so-called enhanced permeation and retention (EPR) effect resulting in higher local drug concentrations occurring in the tumor. ${ }^{25}$

To gain insight into the side effects of NMPs, we made a comparison of the side effects of nab-paclitaxel and conventional paclitaxel. Table 4 presents an overview of the differences in type or frequency between the observed side effects of nab-paclitaxel (as reported for the brand name Abraxane $^{\circledR}$ ) and conventional paclitaxel (as reported for the brand name Taxol ${ }^{\circledR}$ ). Besides the side effects observed with the same frequency, including neutropenia, gastrointestinal disorders, peripheral neuropathy (nerve damage including damage to the nerves in the hand and feet), arthralgia, and myalgia, which are occurring commonly or very commonly, and side effects observed with a different frequency for both drugs, 165 side effects were described specifically for nab-paclitaxel and 55 side effects specifically for conventional paclitaxel. Most of the specific side effects $(\geq 5$ very common and common side effects) of nab-paclitaxel, other than conventional paclitaxel, are observed in the SOCs: nervous system disorders; eye disorders; respiratory, thoracic, and mediastinal disorders; gastrointestinal disorders; skin and subcutaneous tissue disorders; and the SOC investigations.

With respect to the cases in the Lareb database (Supplementary materials), relatively more cases of hepatobiliary disorders, hematological investigations, and neoplasms (benign, malignant, and unspecified, including cysts and polyps) are reported for nab-paclitaxel compared to conventional paclitaxel. The hepatobiliary disorders mentioned for nab-paclitaxel (each $n=1$ ) are not well documented and consist of biliary structure, biliary disorder (not otherwise specified), cholecystitis, and liver disorder (not otherwise specified). The relatively larger difference in occurrence of neoplasms for nabpaclitaxel compared to conventional paclitaxel is caused by five reports of pancreatic cancer for nab-paclitaxel. Although reported as side effect, nab-paclitaxel was in fact prescribed for pancreatic cancer, and this is considered a progressive disease. The reporting of a disease as side effect stems from the obligation for manufacturers to report such cases, because it may indicate lack of effectiveness of the drug. However, 


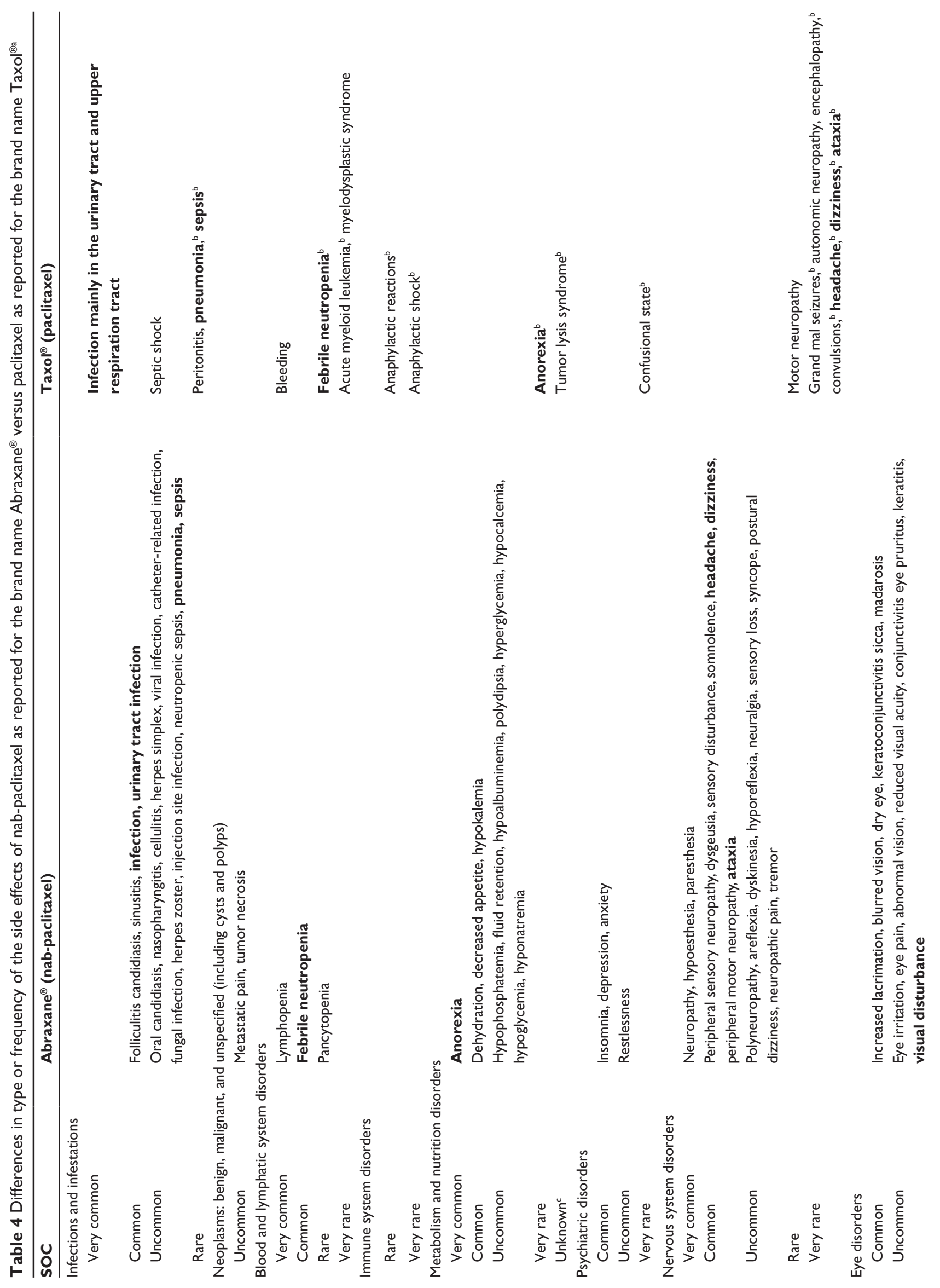



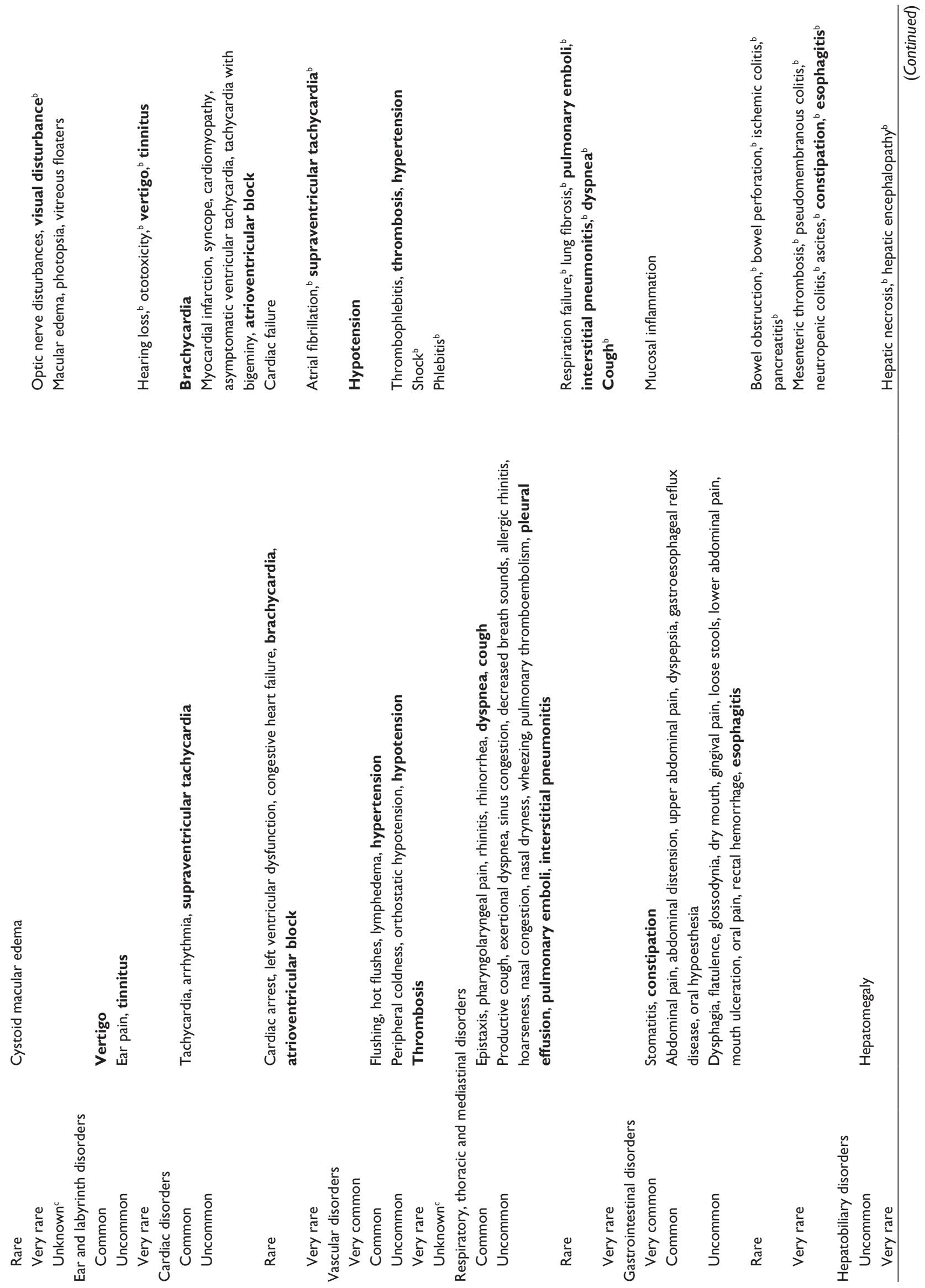

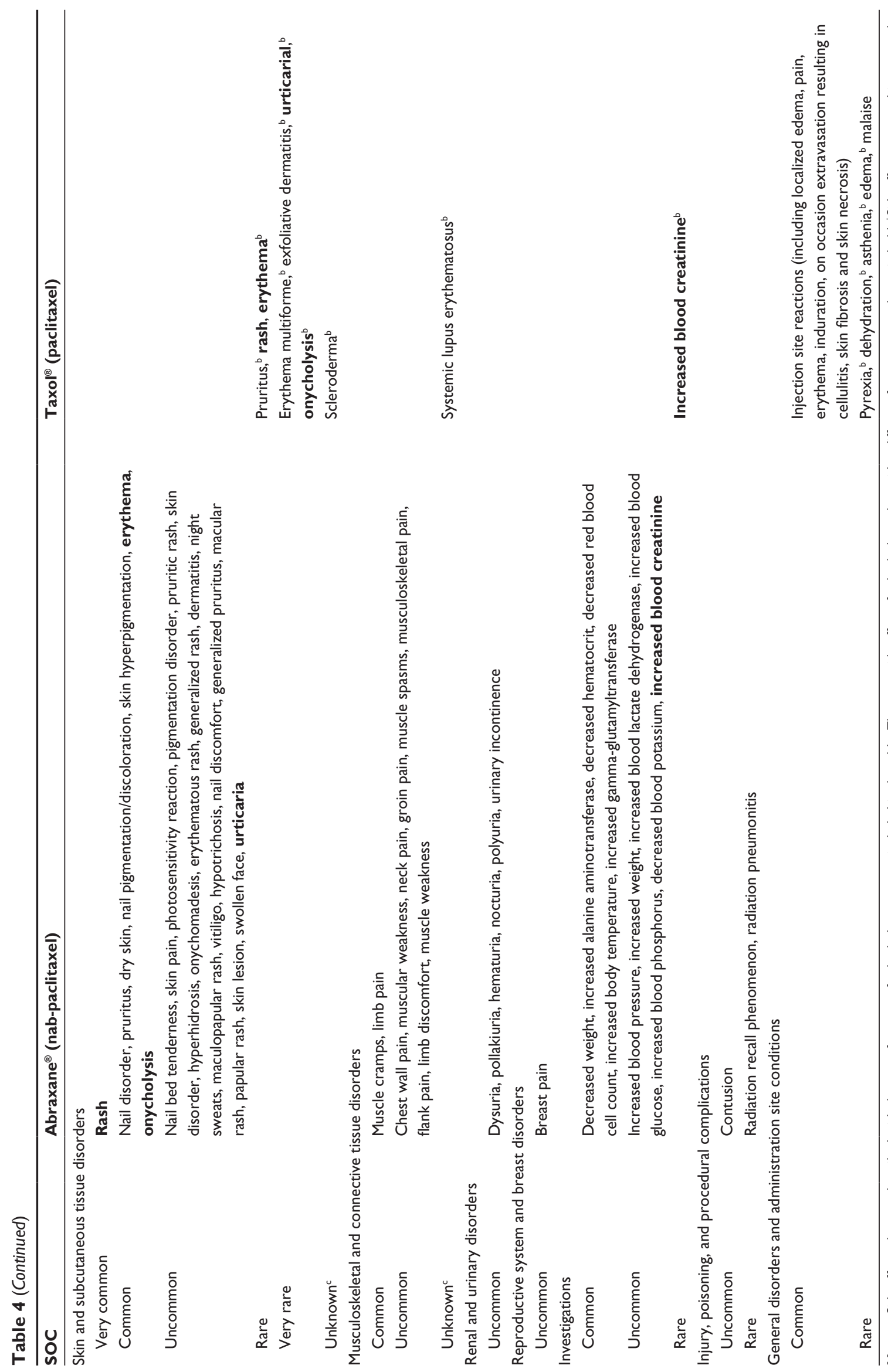


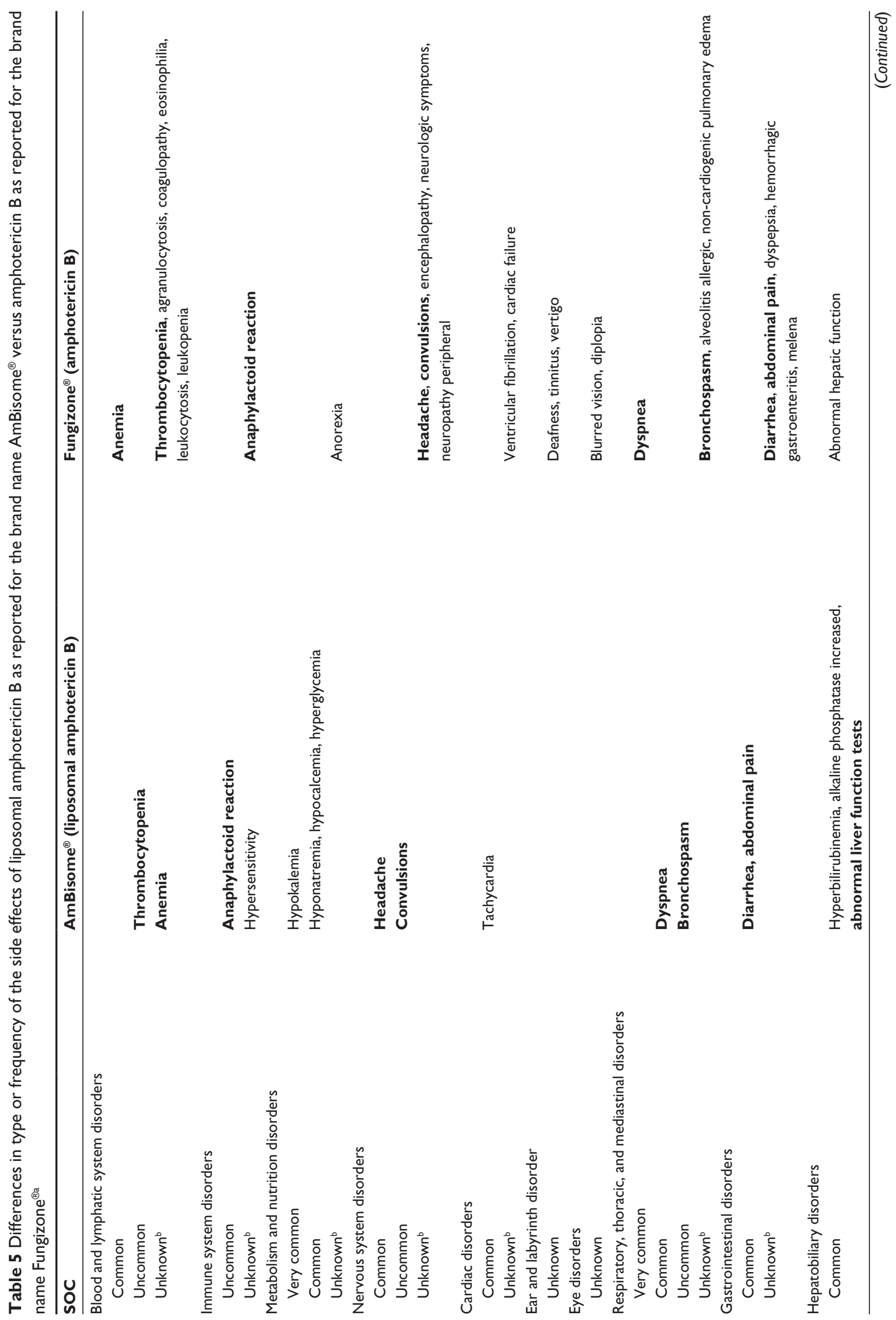




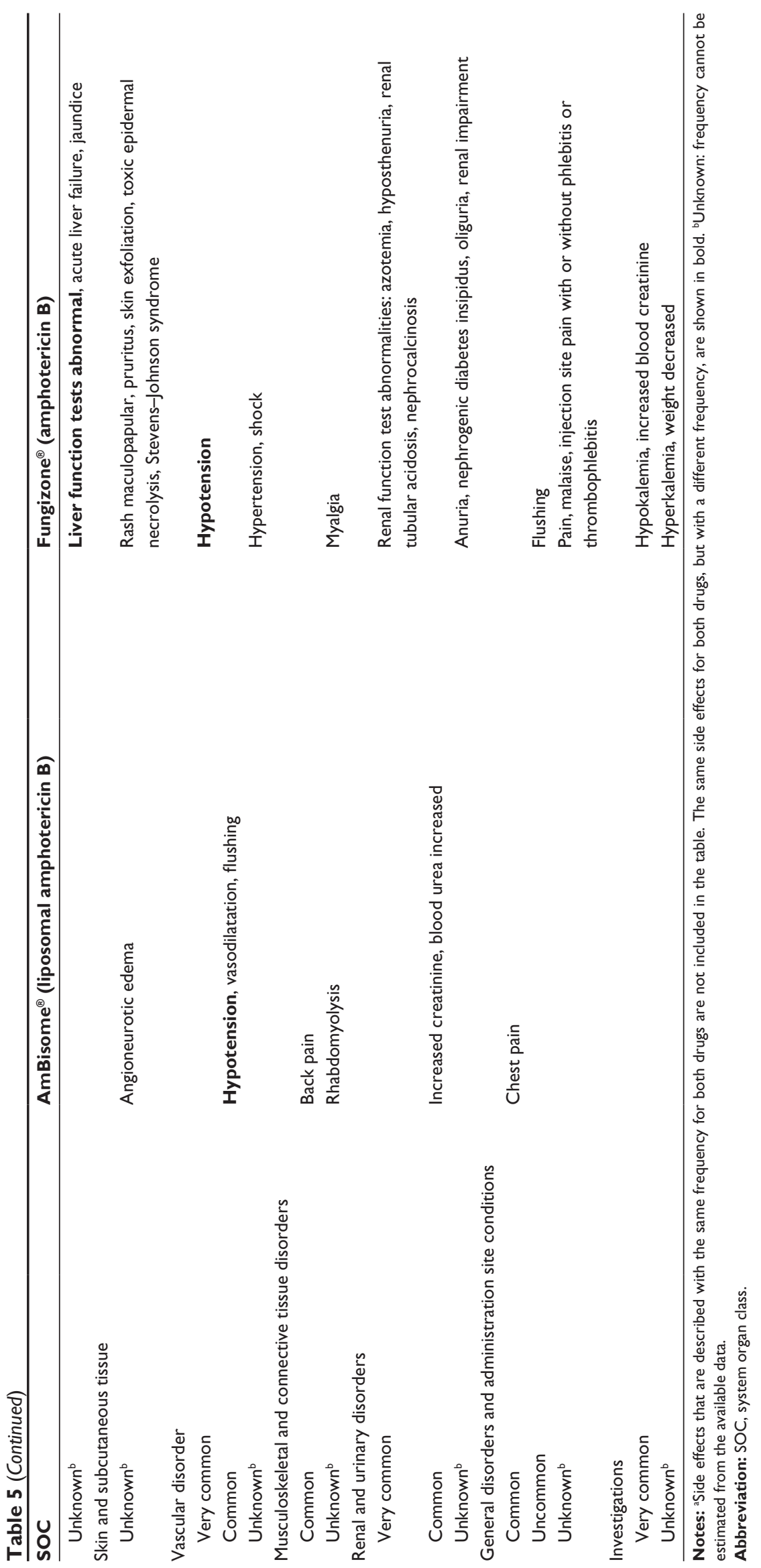




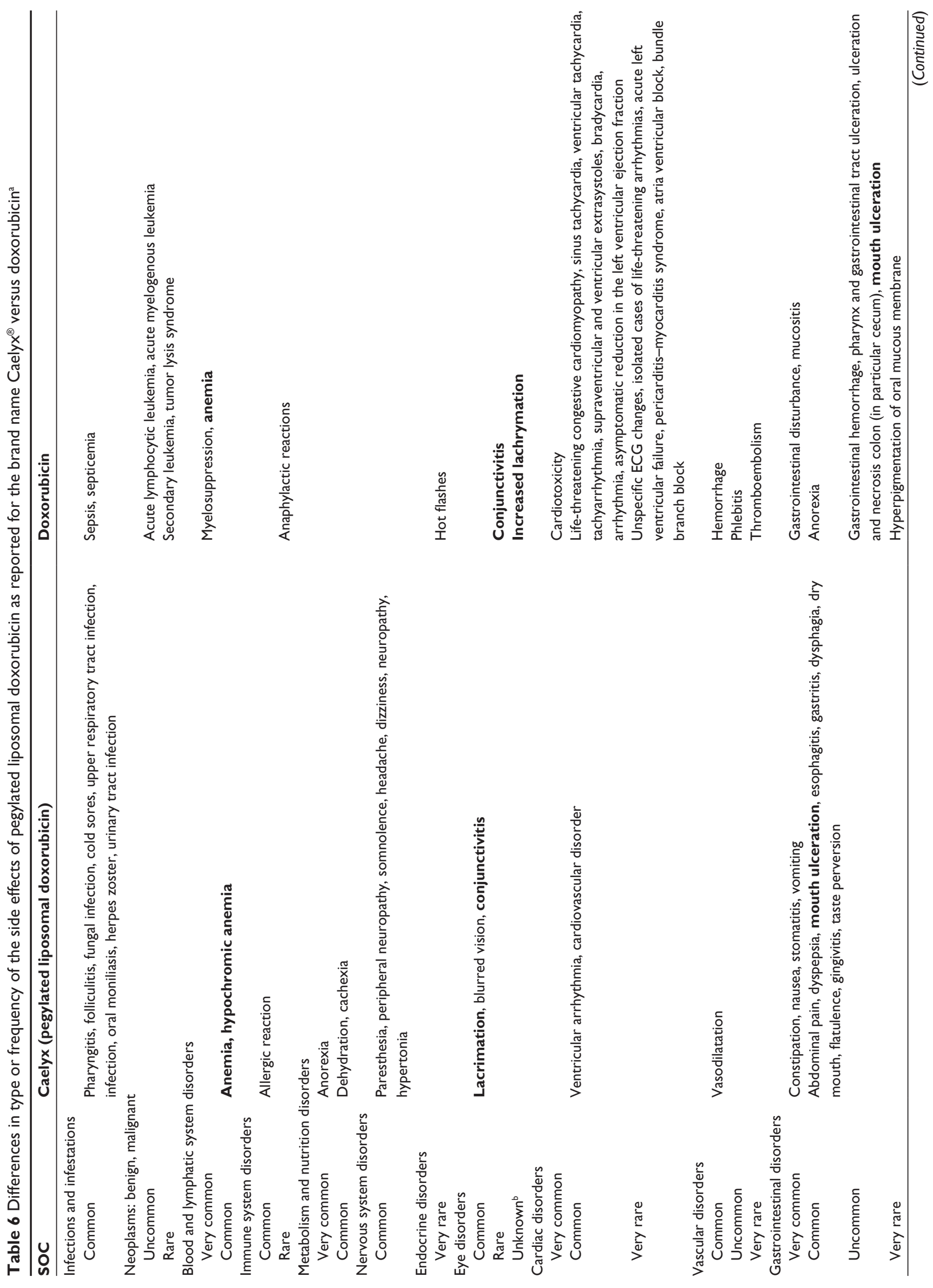




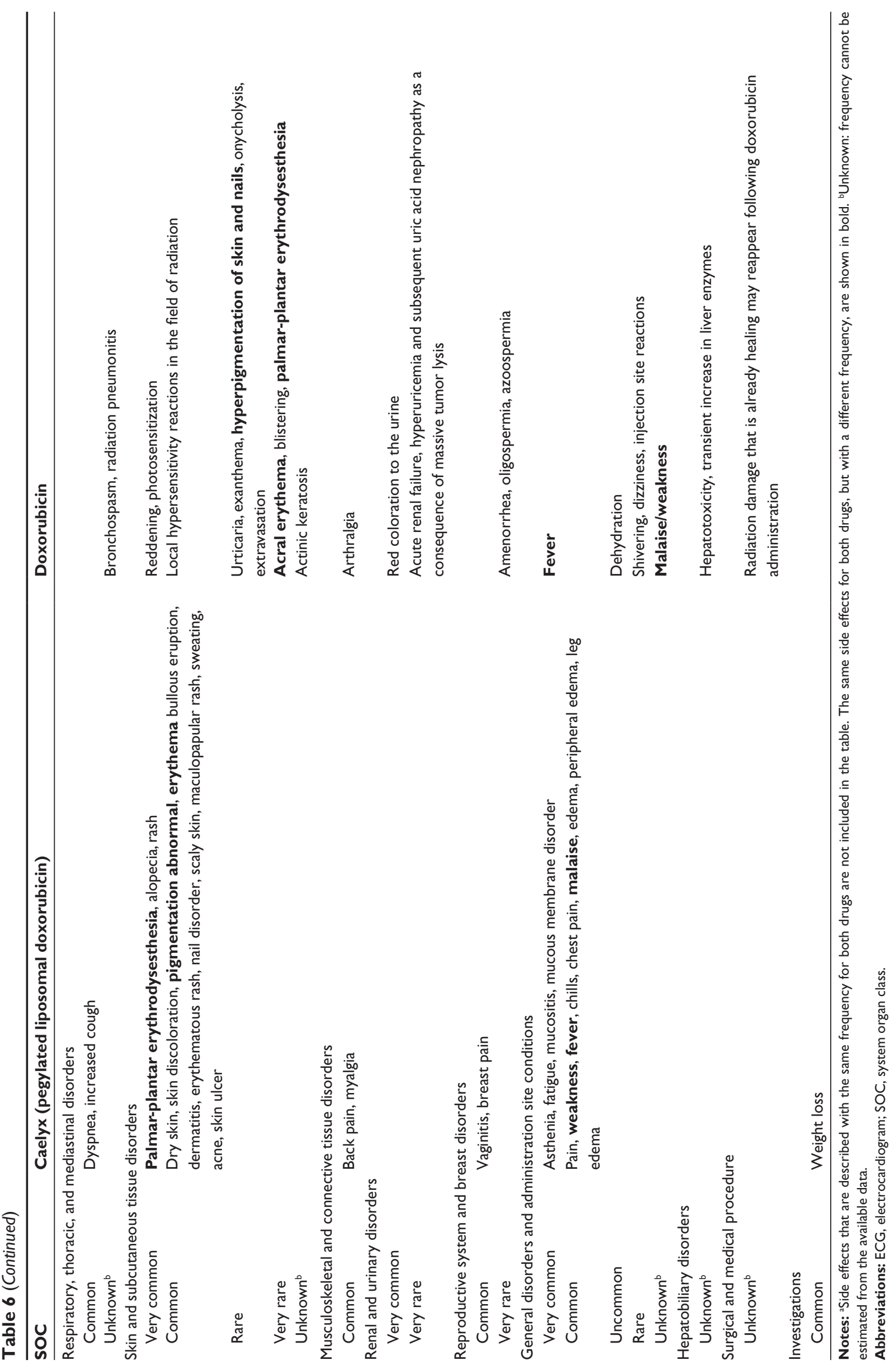




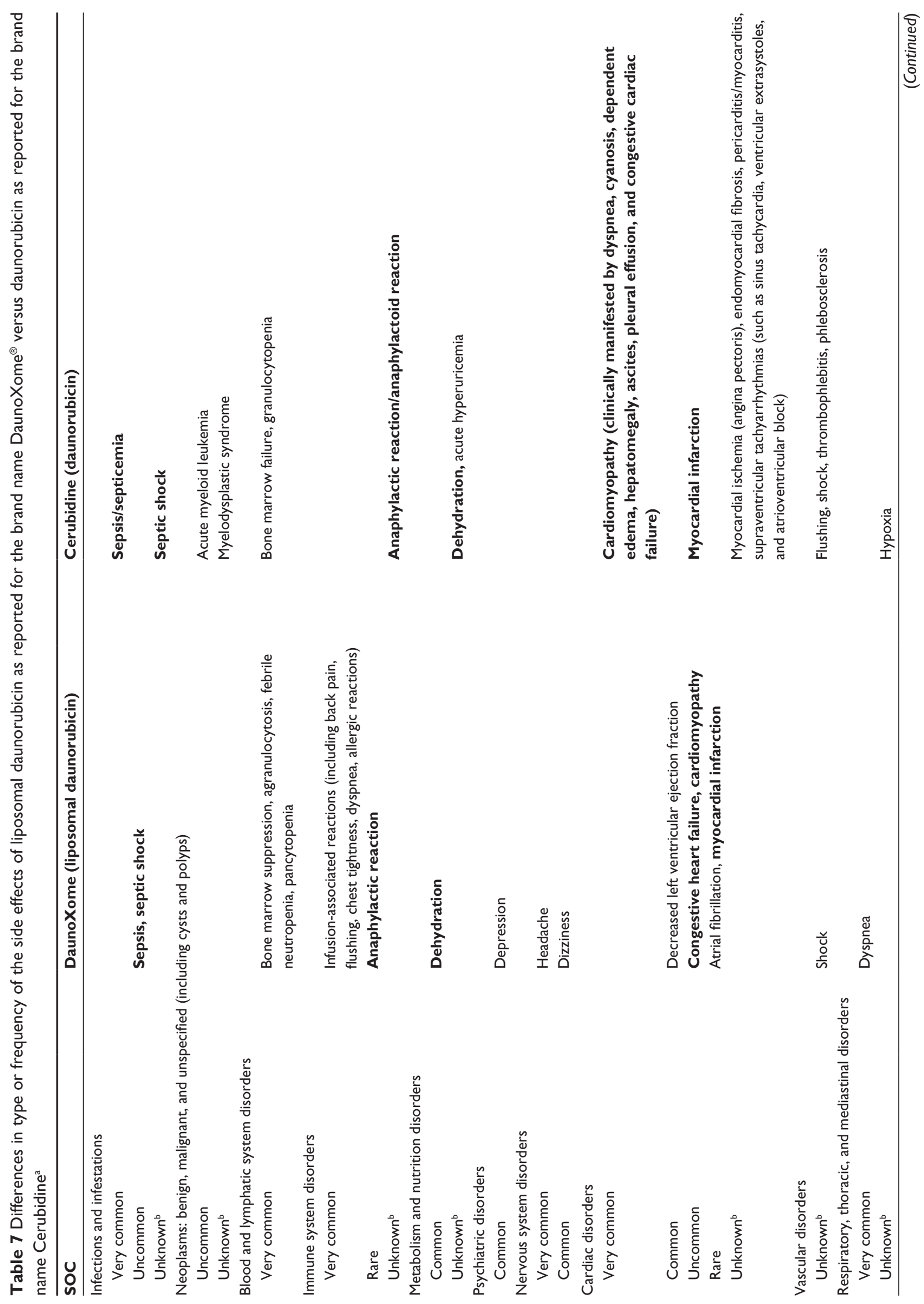




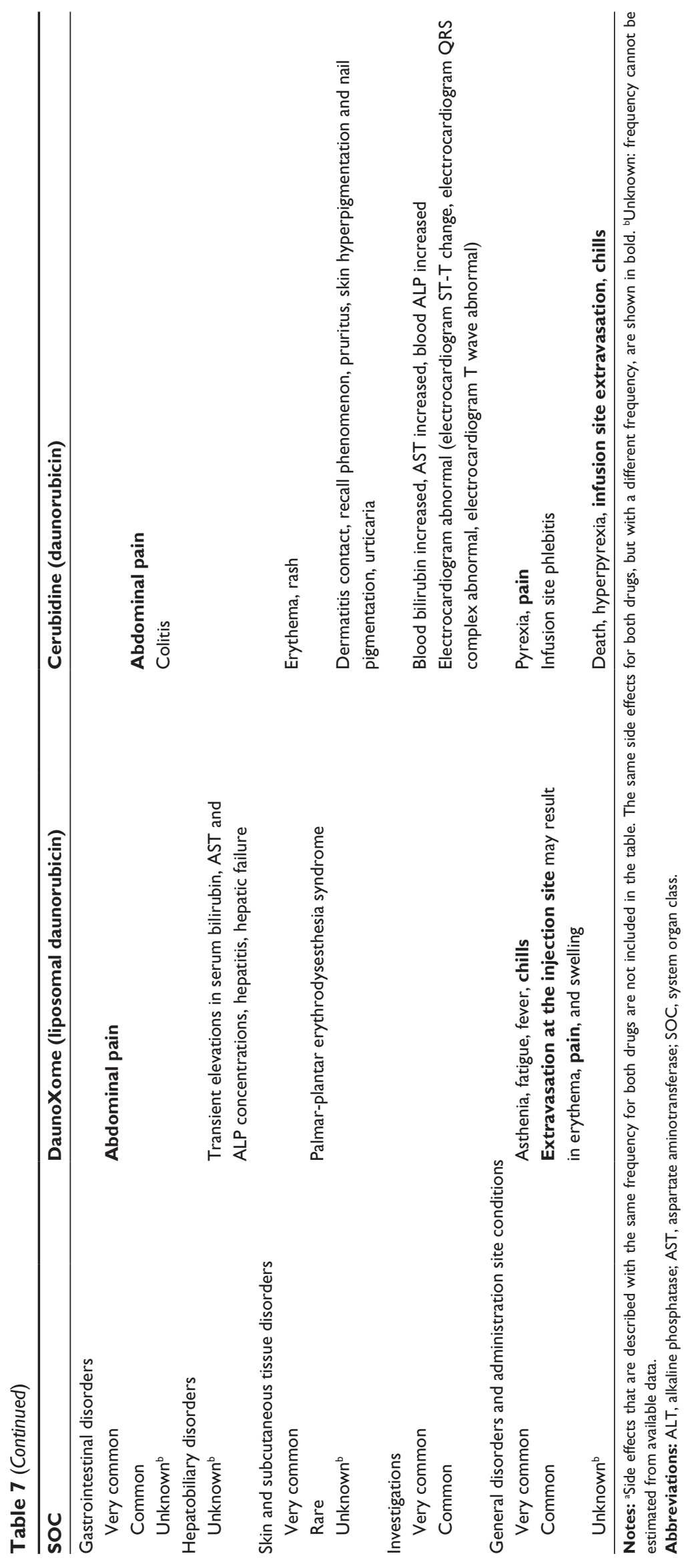


Table 8 Differences in type or frequency of the side effects of liposomal cytarabine as reported for the brand name DepoCyt ${ }^{\circledR}$ versus Cytarabine ${ }^{\circledR a}$

\begin{tabular}{lll}
\hline SOC & DepoCyt (liposomal cytarabine) & Cytarabine \\
\hline $\begin{array}{l}\text { Nervous system disorders } \\
\text { Very common Confusion }\end{array}$ & \\
Common & Confusion \\
$\begin{array}{l}\text { General disorders and administration site conditions } \\
\text { Very common }\end{array}$ & Fatigue \\
Common $\quad$ Fatigue & \\
\hline
\end{tabular}

Notes: aside effects that are described with the same frequency for both drugs are not included in the table. The same side effects for both drugs, but with a different frequency, are shown in bold.

Abbreviation: SOC, system organ class.

disease progression eventually occurs in most patients, so this may not be regarded in this paper as a true side effect.

Overall, nab-paclitaxel is associated with more and different side effects than conventional paclitaxel (Table 4). However, the side effects reported could in this case very well originate from the situation in which nab-paclitaxel and conventional paclitaxel are used, in different treatment regimens or for different cancers as their indications are different, ${ }^{26}$ which is also reflected by the indicative information from Lareb (Supplementary materials). Regarding efficacy, nab-paclitaxel is reported by the EMA to be more effective than conventional paclitaxelcontaining medicines in patients with metastatic breast cancer whose first treatment had stopped working. ${ }^{27}$ An advantage of the use of nab-paclitaxel is that, unlike most other conventional paclitaxel-containing medicines needing solvents such as polyethoxylated castor oil and dehydrated ethanol, patients do not need pretreatment with other medicines such as antihistamines and corticosteroids to prevent hypersensitivity reactions related to these solvents. ${ }^{27,28}$

\section{Liposomal amphotericin B versus amphotericin B}

Amphotericin B is a polyene antibiotic, widely used against life-threatening systemic infections with fungi such as Candida albicans or Aspergillus fumigates as well as parasites such as Leishmania donovani. Amphotericin B is an effective therapeutic, although acute or infusionrelated toxicity and chronic nephrotoxicity was observed. ${ }^{29}$ These toxicities can be reduced by the incorporation of amphotericin B into a lipid-based carrier system, which alters the pharmacokinetics and tissue distribution of the drug. Different formulations have been used to minimize nephrotoxicity including liposomal amphotericin B. Liposomal amphotericin B has proven to reduce the toxicities associated with the administration of the conventional deoxycholate formulation of amphotericin B and to have efficacy against various fungi. ${ }^{30}$ The incorporation of amphotericin $\mathrm{B}$ into a liposome provides the toxicokinetic and mechanistic basis for the increased safety and tolerability. ${ }^{30}$ Liposomal amphotericin B is intended for intravenous administration only, while conventional amphotericin $\mathrm{B}$, is also available for oral administration.

Table 5 presents an overview of the differences in type or frequency between the observed side effects of liposomal amphotericin B (as reported for the brand name AmBisome ${ }^{\circledR}$ ) and conventional amphotericin B (as reported for the brand name Fungizone ${ }^{\circledR}$ ). Besides the side effects observed with the same frequency for both drugs, and side effects observed with a different frequency for both drugs, 16 side effects were described specifically for liposomal amphotericin B and 52 specifically for amphotericin B. Only a few side effects of liposomal amphotericin B, other than conventional amphotericin B, were observed very commonly or commonly (Table 5).

With respect to the cases in the Lareb database, relatively more general disorders and administration site conditions were reported for liposomal amphotericin B. This might be due to the method of administration or due to the reported lack of efficacy; however, the absolute numbers are low (Supplementary materials).

In line with the difference in side effects (Table 5), in the literature, liposomal amphotericin B is reported to induce significantly less nephrotoxicity than conventional amphotericin B and also fewer infusion-related reactions. ${ }^{31,32}$ The higher infusion rate of liposomal amphotericin B is also helpful in this respect. ${ }^{33}$

\section{Pegylated liposomal doxorubicin versus doxorubicin}

Doxorubicin, also known as hydroxydaunorubicin, is a drug used in chemotherapy. It is an anthracycline antibiotic, which is commonly used in the treatment of a wide range of cancers, including hematological malignancies, many types of carcinoma, and soft tissue sarcomas.

A PEGylated (polyethylene glycol [PEG] coated) liposome-encapsulated form of doxorubicin substantially extends the half-life of doxorubicin in vivo. ${ }^{34}$ The effect of extending half-life is attributed to the protection of the liposome by the surface coating of PEG molecules. Both regular doxorubicin and pegylated liposomal doxorubicin are intended for intravenous administration.

Table 6 presents an overview of the differences in type or frequency between the observed side effects of pegylated liposomal doxorubicin (as reported for the brand name Caelyx) 
and regular doxorubicin (as reported for doxorubicin). The most severe side effect of doxorubicin is cardiomyopathy limiting the maximum cumulative dose that can be used to treat a patient as it results in congestive heart failure. Besides the side effects observed for both drugs, 66 side effects were described specifically for pegylated liposomal doxorubicin and 59 side effects specifically for conventional doxorubicin (Table 6). Most of the specific side effects ( $>5$ very common and common side effects) of pegylated liposomal doxorubicin were observed in the SOCs: infections and infestations, nervous system disorders, gastrointestinal disorders, skin and subcutaneous tissue disorders, and general disorders. Most of the specific side effects of conventional doxorubicin were cardiac disorders.

According to the cases in the Lareb database, relatively more immune system disorders; nervous system disorders; respiratory, thoracic, and mediastinal disorders; and skin and subcutaneous tissue disorders were recorded for pegylated liposomal doxorubicin compared to conventional doxorubicin (Supplementary materials). The difference in immune system disorders between the two types of drugs, although not statistically significant, is consistent with the information in Table 6 , ie, common allergic reactions for pegylated liposomal doxorubicin compared to only rare anaphylactic reactions for conventional doxorubicin. In addition, the difference in the SOC nervous system disorders; respiratory, thoracic, and mediastinal disorders; and skin and subcutaneous tissue disorders between the two types of drugs is consistent with the side effects in Table 6. The difference in reporting between pegylated liposomal and conventional doxorubicin is mainly caused by the hand-foot syndrome (palmar-plantar erythrodysesthesia) including neuropathy, which is a side effect of multiple chemotherapeutic agents, but more so for PEG-modified liposomal chemotherapeutic preparations. ${ }^{35}$

Overall, there are certain specific side effects associated with pegylated doxorubicin compared to regular doxorubicin, of which some can be related to the properties of the nanoformulation. In addition to the hand-foot syndrome for PEG-modified chemotherapeutics mentioned earlier, pegylated therapeutics can also cause unexpected immunemediated side effects. ${ }^{36}$ Interestingly, immunotoxicity has not been studied preclinically for pegylated liposomal doxorubicin. With respect to efficacy, in first-line therapy for metastatic breast cancer, pegylated doxorubicin provides comparable efficacy to regular doxorubicin, with significantly reduced cardiotoxicity, myelosuppression, vomiting, and alopecia. ${ }^{37}$

\section{Liposomal daunorubicin versus daunorubicin}

Daunorubicin, also known as daunomycin, is a chemotherapeutic drug that is given as a treatment for some types of cancer, especially specific types of leukemia. In addition to daunorubicin hydrochloride, daunorubicin is also available as a liposomal formulation, which has been shown to result in higher blood levels because of slower distribution and degradation. ${ }^{38,39}$ As a result, it could be more successful in specific treatment regimens. ${ }^{40}$ Both liposomal daunorubicin and regular daunorubicin are intended for intravenous administration.

Table 7 presents an overview of the differences in type or frequency between the observed side effects of liposomal daunorubicin (as has been reported for the brand name DaunoXome ${ }^{\mathbb{R}}$ ) and conventional daunorubicin (as reported for the brand name Cerubidine ${ }^{\circledR}$ ). Besides the side effects observed for both drugs, 22 side effects were described specifically for liposomal daunorubicin and 29 side effects specifically for conventional daunorubicin (Table 7). The very common side effects specific for liposomal daunorubicin that were observed in the SOCs are immune system disorders, nervous system disorders, general disorders, and administration site conditions. The SOC immune system disorders include very common infusion-associated reactions including back pain, flushing, chest tightness, dyspnea, and allergic reactions, as well as rare anaphylactic reactions for liposomal daunorubicin. Anaphylactic reactions are also among the side effects of conventional daunorubicin, although the frequency of these is unknown. During the preclinical studies, immunotoxicity has not been specifically studied for liposomal daunorubicin. Liposomal daunorubicin is associated with reduced cardiotoxicity compared to conventional daunorubicin, ${ }^{15}$ which is also represented by the results in Table 7 .

A comparison of the pharmacovigilance data for both products could not be made, as no reports were available on liposomal daunorubicin in the Lareb database, probably because in the Netherlands liposomal daunorubicin is only approved to treat AIDS-related Kaposi's sarcoma. This aspect would anyway hamper the comparison of spontaneously reported side effects.

\section{Liposomal cytarabine versus cytarabine}

Cytarabine, also known as cytosine arabinoside (ara-C), is a chemotherapy agent used mainly in the treatment of cancers of white blood cells such as acute myeloid leukemia and non-Hodgkin lymphoma. ${ }^{13,41}$ Liposomal cytarabine is a slowrelease cytarabine formulation that encapsulates the drug in spherical multivesicular particles resulting in a gradual 
release into the cerebrospinal fluid. This increases the cerebrospinal fluid half-life of the drug, with overall exposure per injection about 40 times higher than with conventional cytarabine. ${ }^{41}$ Conventional cytarabine is intended for intravenous or subcutaneous administration, while liposomal cytarabine is intrathecally administered in the cerebrospinal fluid of the central nervous system.

Table 8 presents an overview of the differences in type or frequency of the observed side effects between liposomal (as reported for the brand name DepoCyt ${ }^{\circledR}$ ) and conventional cytarabine. Besides side effects observed with the same frequency for both drugs, including headache, arachnoiditis, confusion, nausea, vomiting, diarrhea, pyrexia, weakness, and thrombocytopenia which are occurring commonly or very commonly, no side effects were described specifically for liposomal cytarabine or conventional cytarabine. Only two side effects were described with a different frequency: confusion and fatigue (Table 8), which can be regarded as a minor difference.

Only a limited number of reports for liposomal cytarabine can be found in the Lareb database, and this drug is registered for a different indication (intrathecal treatment of lymphomatous meningitis) than other cytarabine products, which hampers comparison (Supplementary materials).

Liposomal cytarabine has shown similar activity as regular, free cytarabine, with a lower toxicity and a more convenient dosing regimen in the treatment of both lymphomatous meningitis and meningitis from solid tumors. ${ }^{13,14}$ However, the lower toxicity of liposomal cytarabine could not be confirmed based on the comparison of the types or frequencies of the side effects when compared to conventional cytarabine.

\section{Discussion}

In scientific literature, examples are reported where the switch to a nanoformulation of a drug resulted in changed pharmacokinetics and a subsequent shift in toxicity profile. ${ }^{13,15,37,41}$ Nonetheless, the nanoformulation may reduce the overall toxicity of a drug, the severity of the side effects, or better suit specific treatments of patients. We wished to evaluate whether specific end points of toxicity require specific or further attention with respect to NMPs. To get better insight into nanospecific effects from the available data, we analyzed publicly available preclinical toxicity data of 29 NMPs and made comparisons of registered side effects for five sets of two formulations of the same API: one nanoformulation and one conventional formulation. To our knowledge, this is the first time that such an analysis has been performed.
With regard to the preclinical toxicity data, sometimes data on specific end points were not available, or tests were not performed, though often with good reason. For example, when no data are reported on acute toxicity in the preclinical information, this is often because of reference to a concept paper on single-dose/acute toxicity by the EMA Committee for Medicinal Products for Human Use (CHMP) ${ }^{10}$ and additional reference to the repeated-dose toxicity studies. The currently, revised guidelines removed the need for single-dose studies, as often the required data can be derived from the repeated-dose study. ${ }^{42}$ Another reason for not performing an acute toxicity assay is the known acute toxicity of the API (in case of cytostatics) or the known effects of a similar product. This can be a reason for omitting testing for subacute toxicity (repeated dose). Similarly, reproductive toxicity and developmental toxicity testing has been waived because of the known reproductive toxicity, teratogenicity, or developmental effects of the API. In preclinical toxicity data, reproductive toxicity and developmental toxicity are sometimes mentioned together, as both separate end points can be studied within the same assay (eg, in the extended onegeneration assay [Organization for Economic Co-operation and Development Test Guideline 443]). Overall, with regard to the availability of data, these are limitations because of the use of preclinical study information. Data on some toxicological end points seemed scarce compared to other end points; especially little information on carcinogenicity and immunotoxicity was available in the preclinical study information. This was the case for all three groups (Table 2).

Data on carcinogenicity are often not required for registration of a medicine depending on the nature or the intended use of the medicine or can be waived because of the known carcinogenicity of the API. Carcinogenicity studies should be performed for pharmaceuticals whose expected clinical use is continuously for at least half a year, or if there is concern about the carcinogenic potential. ${ }^{43}$ For many NMPs, eg, verteporfin, pegfilgrastim $\left(\right.$ Neulasta $\left.^{\circledR}\right)$, ferumoxytol, or caspofungin acetate $\left(\right.$ Cancidas $\left.^{\circledR}\right)$, human exposure is not long or frequent enough to require the performance of carcinogenicity tests.

For immunotoxicity, the International Council for Harmonization of Technical Requirements for Pharmaceuticals for Human Use S8 guideline provides a decision tree to design an immunotoxicity testing strategy and describes nonclinical assays that can be used for this purpose ${ }^{44}$ Initial information on immunotoxicity comes from the repeateddose toxicity study and is based on a limited number of immune-related parameters, such as specific hematological 
changes, alterations in immune system organ weights, and/or histology. Depending on the outcome of these parameters, additional more specific immunotoxicity studies can be performed. However, it is uncertain whether this procedure provides sufficient information for an adequate evaluation of potential immunotoxicity of NMPs. ${ }^{45}$ Compared to small molecular entities, the immune system acts to eliminate or interact with NPs to a greater extent, and therefore, immunotoxicity is an end point of specific interest with respect to NP-specific toxicity. ${ }^{46,47}$ The accumulation of iron NPs in organs of the reticuloendothelium system (eg, the spleen), for instance, can induce adverse health effects by disturbing immune homeostasis, ${ }^{48}$ and the formation of protein coronas could modulate the immune response. ${ }^{49}$ A recent comparison between current regulatory testing requirements and the accumulating knowledge on immunotoxic effects of NMPs showed that specific immunotoxic effects associated with NMPs, such as complement activation-related pseudo-allergy (CARPA), myelosuppression, inflammasome activation, and hypersensitivity, are not readily detected by using current testing guidelines. ${ }^{45}$

Our first objective was to investigate whether specific toxicity could be related to certain structural types of NMPs. Overall, the data from our survey on the toxicity of NMPs do not indicate that certain toxic effects, in terms of specific end points, are associated with specific groups or structural types of NMPs; however, the numbers of NMPs analyzed per group of NMPs were small. Currently, iron NP-related toxicity and NMP-induced CARPA are the best known "nanospecific" types of toxicity of NMPs. The iron NP-related toxicity is associated with accumulation of iron NPs in the immune system. ${ }^{19,20}$ The induction of CARPA that has been observed during the first-time treatments with liposomal formulations, and testing for CARPA, is now recommended in bioequivalence evaluations of generic liposomal drug candidates. ${ }^{50}$ In the case of the cytostatics and liposomes in our survey, most of the toxic effects can be attributed to the API rather than to the nanospecific structure of these NMPs. The apparent similarity in general toxicological profiles for other structural groups differs too much or is too nonspecific to draw conclusions for the specific nanostructural group. However, in addition to the limited number of products, also considering the limited data on several toxicological end points, conclusions from this survey with regard to potential (lack of) nanomedicinespecific effects need to be considered carefully. A warrant for specific attention with respect to immunotoxicological properties of nanomedicines may not be supported by specific data from our survey of preclinical data; however, this could be due to the limited dataset available. Nanomaterials have been repeatedly shown to distribute to organs of the immune system and may interact with the immune system. Therefore, we still call for special attention for this end point.

Our second objective was to investigate whether a nanoformulation of an API altered the nature of side effects of the product in humans compared to a conventional formulation. Therefore, a comparison of registered side effects between nanoformulations and conventional formulations of the same API has been performed. It needs to be noted that any differences in treatment or dosing regimens are not taken into account in these data, although these can be the cause of certain differences. It should also be realized that the nanoformulation compared to the regular formulation may change the pharmacokinetic profile of the drug after administration, and therefore also the toxicokinetics. ${ }^{7}$ Four of the five sets concerned liposomal formulations, and four of the five sets concerned an application in cancer treatment (chemotherapy) (Table 3). While comparing nab-paclitaxel and conventional paclitaxel, nab-paclitaxel has more reported side effects than paclitaxel. These could be related to the different cancer treatment regimes in which these products are used. While comparing liposomal amphotericin B and conventional amphotericin B, liposomal amphotericin B shows a considerably lower number of side effects compared to conventional amphotericin B, a finding which has been reported previously. ${ }^{33}$ For doxorubicin, daunorubicin, and cytarabine, a similar number of side effects can be contributed to the respective liposomal nanoformulations compared to their conventional formulations. These numbers, however, do not provide information on the severity of the side effects. For example, the reduced nephrotoxicity of liposomal amphotericin $\mathrm{B}^{30}$ and the reduced cardiotoxicity of pegylated liposomal doxorubicin ${ }^{37}$ are reflected in the reported side effects, as is the occurrence of hand-foot syndrome because of the pegylation of doxorubicin. ${ }^{35}$ The reduced cardiotoxicity of liposomal daunorubicin has not been confirmed in comparative clinical trials but is supposed to be present based on other liposomal studies for anthracyclines. ${ }^{15}$ The lower toxicological profile of liposomal cytarabine compared to conventional cytarabine could not be confirmed.

For three out of the four nanomedicinal liposome products, liposomal amphotericin B, pegylated liposomal doxorubicin, and liposomal daunorubicin, side effects on the immune system were reported. For nab-paclitaxel, no side effects on the immune system were reported specifically for the nanomedicinal formulation; however, since the nanoformulation is derived from human albumin, this is not 
surprising. In general, from literature, the most frequently reported side effect after injection of a nanotherapeutic agent seems to be immune-mediated side effects such as a hypersensitivity reaction, including the acute hypersensitivity reaction CARPA, which follows up the triggering of the complement activation cascade. ${ }^{51,52} \mathrm{With}$ respect to pegylated nanoformulations, such as pegylated liposomal doxorubicin, there are claims that unexpected immune-mediated effects can be caused by anti-PEG antibodies as well. ${ }^{36}$ The importance of such mechanisms should be further studied, and the immunotoxicological effects of NMPs should be more accurately evaluated by an expanded testing strategy, which is equipped to stratify applicable testing for the various types of NMPs. ${ }^{45}$

Our survey using publicly available toxicity information and comparing five sets of drugs presents a first attempt to obtain insights into the side effects specific for NMPs. As more information will become available, more such comparisons can be made and possibly broader conclusions could be drawn. In addition, a more in-depth analysis is necessary with regard to the severity of the side effects: a reduction in severity of side effects rather than in the frequency might be an important step forward in a treatment. Furthermore, the accuracy of the reported frequencies should be verified. It is important to be aware that there is likely a substantial reporting bias in the side effects: it can be envisioned that physicians do not report side effects that are well known, while a more unexpected side effect is reported at least more frequently. ${ }^{53}$

\section{Conclusion}

The survey of toxicity to investigate whether specific toxicity could be related to certain structural types of NMP data did not reveal nanospecific toxicity that could be related to certain types or structures of NMPs. Therefore, except for some wellknown effects (ie, immunotoxicity by iron NPs and CARPA induction by NMPs), the publicly available preclinical toxicological data evaluated in this survey do not indicate a specific end point, or a specific type of structure of NMPs on which assessors of NMP safety should be focused. However, given the limited data for some of the product groups or toxicological end points in the analysis, conclusions with regard to (a lack of) potential nanomedicine-specific effects need to be considered carefully. In particular, the immunotoxicological properties of NMPs need further attention. The comparison to investigate whether a nanoformulation of an API (mainly liposomes and/or cytostatics) altered the nature of side effects compared to a conventional formulation confirmed the induction of pseudo-allergic responses associated with specific NMPs in the literature (eg, with liposomal doxorubicin, and possibly liposomal daunorubicin). Acute adverse immunological effects (hypersensitivity) are a known safety aspect associated with NMPs, and although physicians generally adapt their treatment protocols to anticipate such responses more insight and a validated test strategy to predict these effects may contribute in enabling the prevention of hypersensitivity responses to NMPs. For recommendations, for immunotoxicity testing of NMPs, we refer to our recent publication by Giannakou et al. ${ }^{45}$

\section{Acknowledgments}

This work was supported by the project E/132058 "Nanomedicine benefits \& risks," funded by the research program "Onderzoeksprogramma Geneesmiddelenketen," a collaboration of Dutch governmental bodies involved in medicine evaluation, authorization, and monitoring. Hedwig Braakhuis (Centre for Health Protection, RIVM), Joke Herremans (Centre for Safety of Substances and Products, RIVM), Jan Willem van der Laan (Section Pharmacology, Toxicology and Pharmacokinetics, Medicines Evaluation Board [CBG-MEB]), and Eugène van Puijenbroek (Lareb) are acknowledged for feedback, critical reading, and reviewing of the manuscript prior to submission.

\section{Disclosure}

The authors report no conflicts of interest in this work.

\section{References}

1. Kim BY, Rutka JT, Chan WC. Nanomedicine. N Engl J Med. 2010; 363(25):2434-2443.

2. Murthy SK. Nanoparticles in modern medicine: state of the art and future challenges. Int J Nanomedicine. 2007;2(2):129-141.

3. Rizzo LY, Theek B, Storm G, Kiessling F, Lammers T. Recent progress in nanomedicine: therapeutic, diagnostic and theranostic applications. Curr Opin Biotechnol. 2013;24(6):1159-1166.

4. Thorley AJ, Tetley TD. New perspectives in nanomedicine. Pharmacol Ther. 2013;140(2):176-185.

5. Garduno-Balderas LG, Urrutia-Ortega IM, Medina-Reyes EI, Chirino YI Difficulties in establishing regulations for engineered nanomaterials and considerations for policy makers: avoiding an unbalance between benefits and risks. J Appl Toxicol. 2015;35(10):1073-1085.

6. Wolfram J, Zhu M, Yang Y, et al. Safety of nanoparticles in medicine. Curr Drug Targets. 2015;16(14):1671-1681.

7. Onoue S, Yamada S, Chan HK. Nanodrugs: pharmacokinetics and safety. Int J Nanomedicine. 2014;9:1025-1037.

8. Noorlander CW, Kooi MW, Oomen AG, Park MV, Vandebriel RJ, Geertsma RE. Horizon scan of nanomedicinal products. Nanomedicine (Lond). 2015;10(10):1599-1608.

9. Vargas HM, Amouzadeh HR, Engwall MJ. Nonclinical strategy considerations for safety pharmacology: evaluation of biopharmaceuticals Expert Opin Drug Saf. 2013;12(1):91-102.

10. EMA. Committee for Medical Products for Human Use (CHMP). Concept Paper on Single Dose/Acute Toxicity. EMEA/CHMP/SWP/ 302413/2008. 2008. 
11. Patil RR, Guhagarkar SA, Devarajan PV. Engineered nanocarriers of doxorubicin: a current update. Crit Rev Ther Drug Carrier Syst. 2008; 25(1):1-61.

12. Alphandery E, Grand-Dewyse P, Lefevre R, Mandawala C, DurandDubief M. Cancer therapy using nanoformulated substances: scientific, regulatory and financial aspects. Expert Rev Anticancer Ther. 2015; 15(10):1233-1255.

13. Spina M, Chimienti E, Martellotta F, et al. Phase 2 study of intrathecal, long-acting liposomal cytarabine in the prophylaxis of lymphomatous meningitis in human immunodeficiency virus-related non-Hodgkin lymphoma. Cancer. 2010;116(6):1495-1501.

14. Rueda Dominguez A, Olmos Hidalgo D, Viciana Garrido R, Torres Sanchez E. Liposomal cytarabine (DepoCyte) for the treatment of neoplastic meningitis. Clin Transl Oncol. 2005;7(6):232-238.

15. Ewer MS, Martin FJ, Henderson C, Shapiro CL, Benjamin RS, Gabizon AA. Cardiac safety of liposomal anthracyclines. Semin Oncol. 2004;31(6 suppl 13):161-181.

16. Gradishar WJ, Tjulandin S, Davidson N, et al. Phase III trial of nanoparticle albumin-bound paclitaxel compared with polyethylated castor oil-based paclitaxel in women with breast cancer. J Clin Oncol. 2005; 23(31):7794-7803.

17. Lee KS, Chung HC, Im SA, et al. Multicenter phase II trial of genexol-PM, a cremophor-free, polymeric micelle formulation of paclitaxel, in patients with metastatic breast cancer. Breast Cancer Res Treat. 2008; 108(2):241-250.

18. European Agency for the Evaluation of Medicinal Products (EMEA). Scientific Discussion for the Approval of Visudyne. 2004. Available from: http://www.ema.europa.eu/docs/en_GB/document_library/ EPAR_-_Scientific_Discussion/human/000305/WC500052403.pdf. Accessed February 8, 2016.

19. Kang H, Mintri S, Menon AV, Lee HY, Choi HS, Kim J. Pharmacokinetics, pharmacodynamics and toxicology of theranostic nanoparticles. Nanoscale. 2015;7(45):18848-18862.

20. Laurent S, Saei AA, Behzadi S, Panahifar A, Mahmoudi M. Superparamagnetic iron oxide nanoparticles for delivery of therapeutic agents: opportunities and challenges. Expert Opin Drug Deliv. 2014;11(9): 1449-1470.

21. Enriquez-Navas PM, Garcia-Martin ML. Application of inorganic nanoparticles for diagnosis based on MRI. In: de la Fuente JM, Grazu V, editors. Nanobiotechnology: Inorganic Nanoparticles vs Organic Nanoparticles. Vol. 4. Elsevier; 2012:233-246.

22. Ma P, Mumper RJ. Paclitaxel nano-delivery systems: a comprehensive review. J Nanomed Nanotechnol. 2013;4(2):1000164.

23. Rowinsky EK, Donehower RC. Paclitaxel (taxol). N Engl J Med. 1995; 332(15):1004-1014.

24. Weiss RB, Donehower RC, Wiernik PH, et al. Hypersensitivity reactions from taxol. J Clin Oncol. 1990;8(7):1263-1268.

25. Bertrand N, Wu J, Xu X, Kamaly N, Farokhzad OC. Cancer nanotechnology: the impact of passive and active targeting in the era of modern cancer biology. Adv Drug Deliv Rev. 2014;66:2-25.

26. Cecco S, Aliberti M, Baldo P, Giacomin E, Leone R. Safety and efficacy evaluation of albumin-bound paclitaxel. Expert Opin Drug Saf. 2014; 13(4):511-520.

27. European Agency for the Evaluation of Medicinal Products (EMEA). Assessment Report for Abraxane. 2007. Available from: http://www. ema.europa.eu/docs/en_GB/document_library/EPAR_-_Public_ assessment_report/human/000778/WC500020433.pdf. Accessed February 8, 2016.

28. Kloover JS, den Bakker MA, Gelderblom H, van Meerbeeck JP. Fatal outcome of a hypersensitivity reaction to paclitaxel: a critical review of premedication regimens. Br J Cancer. 2004;90(2):304-305.

29. Patel PA, Fernandes CB, Pol AS, Patravale VB. Oral amphotericin B: challenges and avenues. Int J Pharm Biosci Technol. 2013;1(1):1-9.

30. Vyas SP, Gupta S. Optimizing efficacy of amphotericin B through nanomodification. Int J Nanomedicine. 2006;1(4):417-432.
31. Botero Aguirre JP, Restrepo Hamid AM. Amphotericin B deoxycholate versus liposomal amphotericin B: effects on kidney function. Cochrane Database Syst Rev. 2015;11:CD010481.

32. Johansen HK, Gotzsche PC. Amphotericin B lipid soluble formulations versus amphotericin B in cancer patients with neutropenia. Cochrane Database Syst Rev. 2014;9:CD000969.

33. Weissig V, Pettinger TK, Murdock N. Nanopharmaceuticals (part 1): products on the market. Int J Nanomedicine. 2014;9:4357-4373.

34. Vail DM, Amantea MA, Colbern GT, Martin FJ, Hilger RA, Working PK. Pegylated liposomal doxorubicin: proof of principle using preclinical animal models and pharmacokinetic studies. Semin Oncol. 2004; 31(6 suppl 13):16-35.

35. Yokomichi N, Nagasawa T, Coler-Reilly A, et al. Pathogenesis of Hand-Foot syndrome induced by PEG-modified liposomal doxorubicin. Hum Cell. 2013;26(1):8-18.

36. Verhoef JJ, Carpenter JF, Anchordoquy TJ, Schellekens H. Potential induction of anti-PEG antibodies and complement activation toward PEGylated therapeutics. Drug Discov Today. 2014;19(12):1945-1952.

37. Rafiyath SM, Rasul M, Lee B, Wei G, Lamba G, Liu D. Comparison of safety and toxicity of liposomal doxorubicin vs. conventional anthracyclines: a meta-analysis. Exp Hematol Oncol. 2012;1(1):10.

38. Bellott R, Auvrignon A, Leblanc T, et al. Pharmacokinetics of liposomal daunorubicin (DaunoXome) during a phase I-II study in children with relapsed acute lymphoblastic leukaemia. Cancer Chemother Pharmacol. 2001;47(1):15-21.

39. Pea F, Russo D, Michieli M, et al. Liposomal daunorubicin plasmatic and renal disposition in patients with acute leukemia. Cancer Chemother Pharmacol. 2000;46(4):279-286.

40. Latagliata R, Breccia M, Fazi P, et al. Liposomal daunorubicin versus standard daunorubicin: long term follow-up of the GIMEMA GSI 103 AMLE randomized trial in patients older than 60 years with acute myelogenous leukaemia. Br J Haematol. 2008;143(5):681-689.

41. Kripp M, Hofheinz RD. Treatment of lymphomatous and leukemic meningitis with liposomal encapsulated cytarabine. Int J Nanomedicine. 2008;3(4):397-401.

42. EMA. Committee for Medicinal Products for Human Use (CHMP). Questions and Answers on the Withdrawal of the "Note for Guidance on Single Dose Toxicity" (EMA/CHMP/SWP/81714/2010). 2010.

43. EMA. ICH Topic S 1 A. The Need for Carcinogenicity Studies of Pharmaceuticals. Note for Guidance on the Need for Carcinogenicity Studies of Pharmaceuticals (CPMP/ICH/140/95). 2006.

44. EMA. ICH Topic S 8 Immunotoxicity for Human Pharmaceuticals. Note for Guidance on Immunotoxicity Studies for Human Pharmaceuticals (CHMP/167235/2004). 2006.

45. Giannakou C, Park MVDZ, de Jong WH, van Loveren H, Vandebriel RJ, Geertsma RE. A comparison of immunotoxic effects of NMPs with regulatory immunotoxicity testing requirements. Int J Nanomedicine. 2016;11:2935-2952.

46. Pedata P, Petrarca C, Garzillo EM, Di Gioacchino M. Immunotoxicological impact of occupational and environmental nanoparticles exposure: the influence of physical, chemical, and combined characteristics of the particles. Int J Immunopathol Pharmacol. 2016;29(3):343-353.

47. Shannahan JH, Brown JM. Engineered nanomaterial exposure and the risk of allergic disease. Curr Opin Allergy Clin Immunol. 2014;14(2):95-99.

48. Park EJ, Kim SW, Yoon C, Kim Y, Kim JS. Disturbance of ion environment and immune regulation following biodistribution of magnetic iron oxide nanoparticles injected intravenously. Toxicol Lett. 2016;243:67-77.

49. Corbo C, Molinaro R, Parodi A, Toledano Furman NE, Salvatore F, Tasciotti E. The impact of nanoparticle protein corona on cytotoxicity, immunotoxicity and target drug delivery. Nanomedicine (Lond). 2016; 11(1):81-100

50. Szebeni J, Storm G. Complement activation as a bioequivalence issue relevant to the development of generic liposomes and other nanoparticulate drugs. Biochem Biophys Res Commun. 2015;468(3):490-497. 
51. Szebeni J. Hemocompatibility testing for nanomedicines and biologicals: predictive assays for complement mediated infusion reactions. Eur J Nanomed. 2012;4(1):33-53.

52. Szebeni J. Complement activation-related pseudoallergy: a stress reaction in blood triggered by nanomedicines and biologicals. Mol Immunol. 2014;61(2):163-173.
53. Roux E, Thiessard F, Fourrier A, Begaud B, Tubert-Bitter P. Evaluation of statistical association measures for the automatic signal generation in pharmacovigilance. IEEE Trans Inf Technol Biomed. 2005; 9(4):518-527.

\section{Publish your work in this journal}

The International Journal of Nanomedicine is an international, peerreviewed journal focusing on the application of nanotechnology in diagnostics, therapeutics, and drug delivery systems throughout the biomedical field. This journal is indexed on PubMed Central, MedLine, CAS, SciSearch $\AA$, Current Contents ${ }^{\circledR} /$ Clinical Medicine,
Journal Citation Reports/Science Edition, EMBase, Scopus and the Elsevier Bibliographic databases. The manuscript management system is completely online and includes a very quick and fair peer-review system, which is all easy to use. Visit http://www.dovepress.com/ testimonials.php to read real quotes from published authors.

Submit your manuscript here: http://www.dovepress.com/international-journal-of-nanomedicine-journal 